\title{
Cadherin-11 mRNA and protein expression in ovarian tumors of different malignancy: No evidence of oncogenic or tumor-suppressive function
}

\author{
CHARLOTTE VON BÜLOW ${ }^{1}$, LETICIA OLIVEIRA-FERRER ${ }^{1}$, THOMAS LÖNING ${ }^{2}$, \\ FABIAN TRILLSCH $^{1}$, SVEN MAHNER $^{1}$ and KARIN MILDE-LANGOSCH ${ }^{1}$ \\ ${ }^{1}$ Department of Gynecology, University Medical Center Hamburg-Eppendorf, D-20246 Hamburg; \\ ${ }^{2}$ Albertinen Pathologie, D-22457 Hamburg, Germany
}

Received March 31, 2015; Accepted May 27, 2015

DOI: $10.3892 / \mathrm{mco} .2015 .593$

\begin{abstract}
Cadherin-11 (CDH11, OB-cadherin) is a mesenchymal cadherin found to be upregulated in various types of tumors and implicated in tumor progression and metastasis. In order to determine the role of $\mathrm{CDH} 11$ expression in ovarian tumors, we performed a combined reverse transcription quantitative polymerase chain reaction (RT-qPCR), western blot analysis and immunohistochemical study on a large cohort of benign, borderline and invasive ovarian tumors. The RT-qPCR and western blot analysis demonstrated that the CDH11 expression was high in benign cystadenomas and decreased with increasing malignancy. This may be explained by the different tumor-stroma ratios, since immunohistochemistry revealed strong staining of stromal cells, particularly vascular smooth muscle cells and endothelial cells, but only weak cytoplasmic or nuclear immunoreactivity of cancer cells. Within the group of invasive carcinomas, high $\mathrm{CDH} 11$ protein expression, as detected by western blot analysis, was found to be significantly correlated with advanced stage and nodal involvement. However, the recurrence-free and overall survival analyses did not reveal any prognostic or predictive significance. In conclusion, in contrast to other tumor types, CDH11 does not play an important role in ovarian cancer progression.
\end{abstract}

\section{Introduction}

Adhesion proteins of the cadherin family are frequently deregulated during cancer growth and progression. The prototype cadherin of epithelial tissues is E-cadherin, a major constituent of adherens junctions, which participates in cell-cell adhe-

Correspondence to: Dr Karin Milde-Langosch, Department of Gynecology, University Medical Center Hamburg-Eppendorf, 52 Martini Street, D-20246 Hamburg, Germany

E-mail:milde@uke.de

Key words: ovarian cancer, cadherin-11, prognosis, western blot analysis, immunohistochemistry, borderline tumors sion and cell polarity, is involved in differentiation and cell signaling and also acts as a tumor suppressor through its negative impact on cell migration and invasion (1). Disruption of E-cadherin function by mutation, promoter hypermethylation or loss of heterozygosity has been frequently reported in lobular breast cancer and other types of tumors. Ovarian carcinomas are an exception, since E-cadherin is expressed in all stages of tumorigenesis, including metastases and tumor cells in effusions $(2,3)$.

Loss of E-cadherin expression is generally accompanied by increased expression of the mesenchymal cadherins $\mathrm{CDH} 2$ (N-cadherin) and/or cadherin-11 (CDH11, OB-cadherin). This 'cadherin switch' is a major characteristic of the epithelial-mesenchymal transition during the progression of a number of tumor types. Carcinomas expressing $\mathrm{N}$-cadherin exhibit reduced adhesion between tumor cells, but are more able to interact with $\mathrm{N}$-cadherin-positive stromal or endothelial cells (4). In addition, $\mathrm{N}$-cadherin activates signaling pathways leading to enhanced cell migration, invasion and survival (5).

CDH11 is normally expressed in mesoderm-derived tissues, particularly osteoblasts, but is also upregulated in epithelial cancer and stromal cells in carcinomas (6). Increased CDH11 expression was reported in brain tumors and prostate cancer, where it leads to preferential metastasis to the bone $(7,8)$, and in highly invasive breast cancer cell lines, where CDH11 was shown to promote motility and invasive potential $(9,10)$. In other tumor entities, such as osteosarcomas, melanomas and head and neck cancers, a tumor-suppressive effect of CDH11 was reported, with decreased expression in metastases compared with that in primary tumors (6).

The currently available information on the role of CDH11 in ovarian cancer is sparse. In a microarray analysis of serous ovarian carcinomas, CDH11 mRNA expression was increased in metastases compared with that in primary tumors (11). In our own experimental study on the role of the transcription factor c-Fos in ovarian cancer cells, c-Fos overexpression resulted in decreased adhesive properties of the tumor cells, accompanied by downregulation of CDH11 and other adhesion proteins (12). In order to further investigate the role of this adhesion protein in ovarian cancer, we analyzed CDH11 mRNA and protein 
expression in tissue samples of ovarian tumors of different histological subtypes.

\section{Materials and methods}

Patients. Tissue samples of 213 patients with epithelial ovarian tumors were included in our western blot analysis, including benign cystadenomas $(n=5)$, borderline tumors $(n=19)$, invasive primary carcinomas $(n=178)$ and recurrent carcinomas $(n=11)$. The cohort characteristics are summarized in Table I. CDH11 mRNA expression was analyzed in 51 samples, including 6 cystadenomas, 20 borderline tumors and 25 invasive primary carcinomas. CDH11 immunohistochemistry was performed on 3 cystadenomas, 12 borderline ovarian tumors and 8 invasive carcinomas. Surgery was performed at the University Medical Centre Hamburg-Eppendorf between 1994 and 2012, or at the Albertinen Hospital in Hamburg, between 2013 and 2014. All the patients provided written informed consent for examining their tissue samples and reviewing their medical records, according to our Investigational Review Board and Ethics Committee guidelines. A detailed database including clinicopathological factors, histological classifications and therapeutic procedures was generated. The clinical outcomes of all the patients were monitored from the date of surgery until December, 2013.

Tissue samples and protein extraction. The tissue samples were collected intraoperatively and were immediately cryoconserved at $-80^{\circ} \mathrm{C}$. In order to assure a tumor cell content of $\geq 70 \%$, every sample was assessed on cryo-cut sections stained with haematoxylin and eosin. If necessary, stromal parts were removed. Approximately $100 \mathrm{mg}$ of tumor tissue were used for protein extraction, which was performed as previously described (13).

Western blot analysis. Western blot analysis was generally performed as previously described (13). Equal amounts of protein $(20 \mu \mathrm{g})$ from each sample were loaded per well and equal loading was verified by immunoblotting with anti-GAPDH antibody (FL335; Santa Cruz, Heidelberg, Germany; 1:5,000). Proteins from a tumor with known moderate CDH11 expression served as control on each gel. Following electrophoresis and blotting to polyvinylidene difluoride membranes, the membranes were stored at $4^{\circ} \mathrm{C}$ in blocking solution. The rabbit anti-OB-cadherin antibody (P707; Cell Signaling Technology Inc., Danvers, MA, USA) was added to this blocking solution to a final concentration of $0.064 \mu \mathrm{g} / \mathrm{ml}$ and incubated overnight. Peroxidase-conjugated anti-rabbit IgG (sc-2054; dilution, 1:8,000; Santa Cruz) served as the secondary antibody and was visualized by chemiluminescence reagent (Super Signal West Pico kit; Pierce, Rockford, IL, USA) on medical X-ray films (Fujifilm Europe, Düsseldorf, Germany). The band intensities were quantified by densitometry (Imaging Densitometer GS-700; BioRad, Munich, Germany) and calculated as \% intensity of the control tumor sample (CT).

RNA extraction, cDNA synthesis and reverse transcription quantitative polymerase chain reaction ( $R T-q P C R)$. RNA extraction from frozen ovarian tumor tissue and subsequent quality analysis were performed as previously described (14). RNA (5 $\mu \mathrm{g})$ was reverse-transcribed using the
Table I. Cohort characteristics of primary carcinomas $(n=178)$.

\begin{tabular}{|c|c|}
\hline Characteristics & No. $(\%)$ \\
\hline \multicolumn{2}{|l|}{ Age (years) } \\
\hline Mean & 59.4 \\
\hline Median & 61.0 \\
\hline Range & $21-90$ \\
\hline \multicolumn{2}{|l|}{ FIGO stage } \\
\hline I & $8(4.5)$ \\
\hline II & $7(3.9)$ \\
\hline III & $126(70.8)$ \\
\hline IV & $31(17.4)$ \\
\hline Unknown & $6(3.4)$ \\
\hline \multicolumn{2}{|l|}{ Grade } \\
\hline 1 & $9(5.1)$ \\
\hline 2 & $46(25.8)$ \\
\hline 3 & $119(66.9)$ \\
\hline Not determined & $4(2.2)$ \\
\hline \multicolumn{2}{|l|}{ Lymph node status } \\
\hline N0 & $45(25.3)$ \\
\hline N1 & $101(56.7)$ \\
\hline NX & $32(18.0)$ \\
\hline \multicolumn{2}{|c|}{ Postoperative residual tumour } \\
\hline Microscopic & $100(56.2)$ \\
\hline$\leq 1 \mathrm{~cm}$ & $29(16.3)$ \\
\hline$>1 \mathrm{~cm}$ & $15(8.4)$ \\
\hline Not determined & $34(19.1)$ \\
\hline \multicolumn{2}{|c|}{ Histological subtype } \\
\hline Serous & $148(83.2)$ \\
\hline Mucinous & $4(2.2)$ \\
\hline Endometroid & $11(6.2)$ \\
\hline Others & $13(7.3)$ \\
\hline Not determined & $2(1.1)$ \\
\hline \multicolumn{2}{|c|}{ Progression-free survival, months } \\
\hline Mean & 28.5 \\
\hline Median & 15.9 \\
\hline Range & $0-176$ \\
\hline \multicolumn{2}{|c|}{ Overall survival, months } \\
\hline Mean & 40.6 \\
\hline Median & 30.4 \\
\hline Range & $1-176$ \\
\hline
\end{tabular}

FIGO, International Federation of Gynecology and Obstetrics.

Maxima First Strand cDNA Synthesis kit (Thermo Fisher Scientific, Pinneberg, Germany) and the obtained cDNA was diluted (1:5) for further RT-qPCR analysis. The following primers were used for amplification of the CDH11 sequences and the housekeeping gene GAPDH: CDH11: forward, 5'-CCCAGTACACGTTGATGCCT-3' and reverse, 5'-GAC GTTCCCACATTGGACCT-3'; GAPDH: forward, 5'-GTCAGT GGTGGACCTGACCT-3' and reverse, 5'-TGCTGTAGC CAAATTCGTTG-3'. RT-qPCR was performed using the 

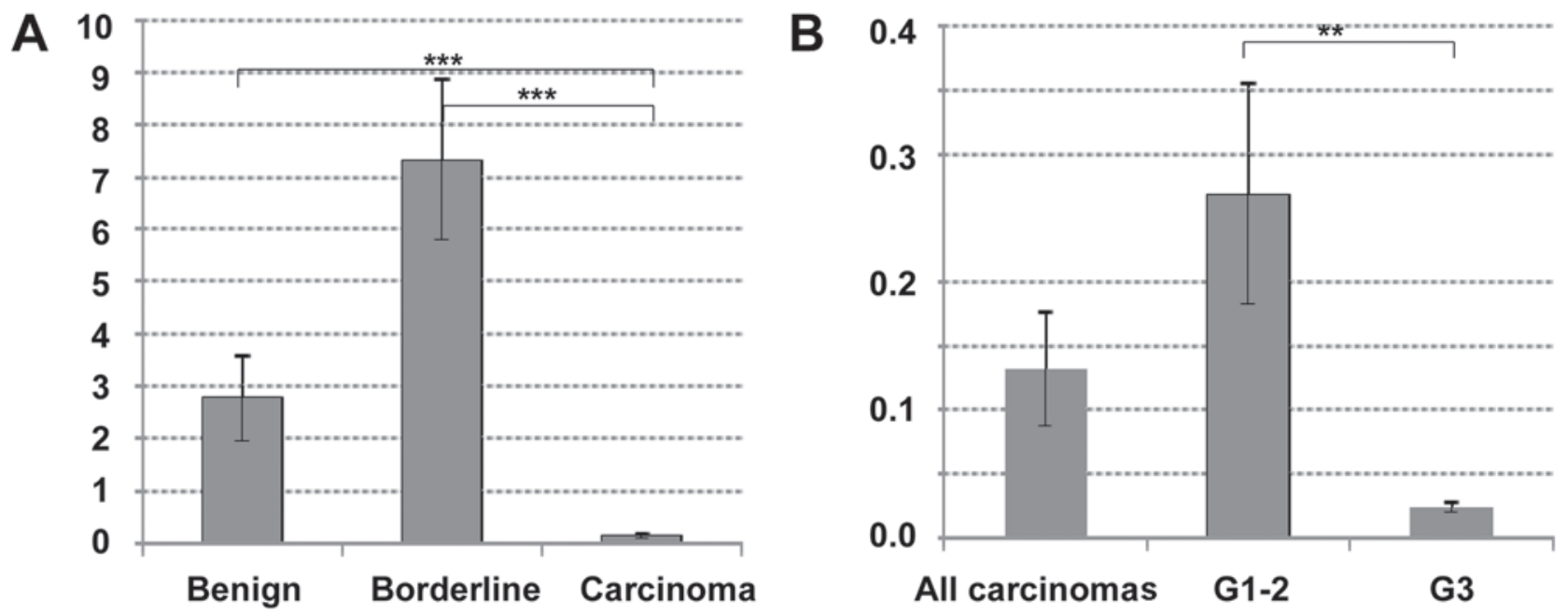

Figure 1. Cadherin-11 mRNA expression in ovarian tumors of different malignancy. (A) Comparison of benign cystadenomas $(\mathrm{n}=6)$, borderline tumors $(\mathrm{n}=20)$ and invasive carcinomas (n=25); (B) comparison of carcinomas of different grading [11 highly/moderately differentiated (grade 1-2) and 14 poorly differentiated (grade 3) carcinomas]. Significant differences following two-sided t-tests are shown by asterisks: ${ }^{* *} \mathrm{P}<0.01$ and ${ }^{* * *} \mathrm{P}<0.00001$.

capillary-based Light Cycler (Roche, Basel, Switzerland) and the SYBR Premix Ex Taq (Takara Bio,Saint-Germain-en-Laye, France) with $1 \mu \mathrm{l}$ cDNA. The samples were analyzed in duplicates and the results were averaged. CDH11 expression was normalized to the reference gene GAPDH and the relative expression of CDH11 in each sample was compared with the expression in one borderline sample, which was used as control (fold change $=1$ ) based on the $\Delta \Delta \mathrm{Ct}$ method.

Immunohistochemistry. For CDH11 immunohistochemistry, paraffin-embedded tissue sections of $4 \mu \mathrm{m}$ were deparafinized and incubated overnight at $4^{\circ} \mathrm{C}$ with the goat polyclonal CDH11 antibody (cat. no. AF1790; R\&D Systems, Abingdon, UK), diluted to 1:150 in antibody diluent (Dako, Glostrup, Denmark) without prior antigen retrieval steps. For detection, the washed slides were incubated with biotin-labelled anti-mouse immunoglobulin (IgG), preformed ABC-Complex (Vectastain; Vector Laboratories, Burlingame, CA, USA) and DAB-substrate kit (Vectastain; Vector Laboratories). All the slides were counterstained with haematoxylin. For negative controls, normal rabbit IgG (Dako) was used instead of primary antibody. The endothelial antigen CD31 was visualized using the monoclonal mouse antibody anti-CD31 (cat. no. M0823; Dako, Hamburg, Germany), diluted 1:60. Images were captured using an AxioVision 40 microscope (Carl Zeiss Imaging Solutions, Munich, Germany) and photoshop software (Adobe Systems Inc., San Jose, CA, USA).

\section{Results}

CDH11 mRNA expression in ovarian tumors of different malignant potential. In a first approach, CDH11 mRNA expression was investigated in 6 cystadenomas/cystadenonofibromas, 20 borderline tumors and 25 invasive ovarian carcinomas. Interestingly, there was a distinct difference between these tumor types, with significantly lower CDH11 expression in carcinomas compared to borderline tumors $(\mathrm{P}<0.00001)$ or cystadenomas $(\mathrm{P}<0.00001)$. There was no statistically significant difference between benign or borderline tumors $(\mathrm{P}=0.14$,
Fig. 1A). Among invasive carcinomas, $\mathrm{CDH} 11$ expression was lower in poorly differentiated tumors (grade 3) compared with that in highly or moderately differentiated carcinomas (grade 1-2, $\mathrm{P}=0.004$, Fig. 1B). No significant correlation to age, stage, nodal involvement or histological tumor type was observed (data not shown).

CDH11 protein expression in ovarian tumors. By western blot analysis, CDH11 was detected in almost all 213 tissue samples in varying amounts (Fig. 2A). Compared to the CT included in all the experiments, the protein expression levels ranged from 0.03 to $400 \%$ (mean, $90.5 \%$; median, $72.0 \%$ ). Regarding the tumor types, the mean CDH11 protein expression decreased with increasing malignancy, being $147 \%$ in benign tumors, $124 \%$ in borderline tumors and $86 \%$ in carcinomas (Fig. 2B). The difference between invasive cancer and borderline tumors was statistically significant $(\mathrm{P}=0.045)$. Recurrent tumors exhibited higher CDH11 protein levels (96\%) compared with primary carcinomas (86\%), but this difference did not reach statistical significance.

In 39 tumor samples representing all tumor types, CDH11 expression was analyzed at the protein and mRNA levels. By the Pearson's correlation test, there was no significant correlation between mRNA and protein data in this sub-cohort $(\mathrm{r}=0.280 ; \mathrm{P}=0.084)$.

Correlations of CDH11 protein expression with clinical or histological parameters and prognosis in ovarian cancer. Based on CDH11 protein expression values, the cohort of 178 primary carcinomas was first divided into 4 quartiles of equal size with low, moderate, strong and very strong CDH11 expression. Comparing these 4 groups by Chi-square tests or using the median value as cut-off, there was no significant association with grade, age, International Federation of Gynecology and Obstetrics stage or lymph node involvement (data not shown). Yet, if the first quartile was compared with the upper 3 quartiles, low CDH11 expression was found to be significantly correlated with early stage and negative lymph node status (Fig. 2C and D). Regarding the histological 

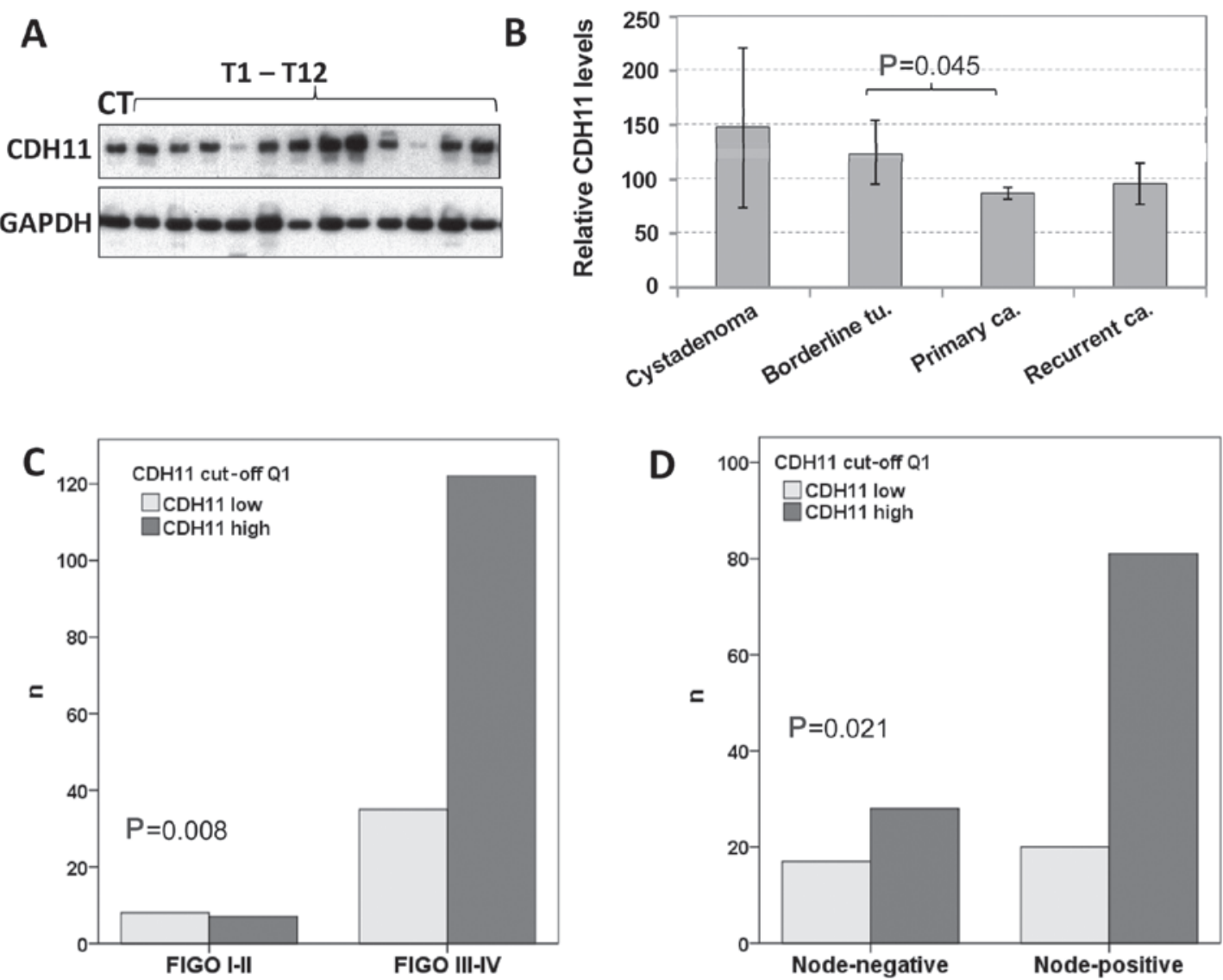

Figure 2. Cadherin-11 (CDH11) protein expression in ovarian tumors of different malignancy. (A) Representative western blot analyses showing CDH11 expression in a control tumor sample (CT) and 12 additional carcinomas. For comparison, expression of the housekeeping gene glycerinaldehyde-3-phosphate dehydroxygenase (GAPDH) is shown; (B) comparison of $\mathrm{CDH} 11$ expression in benign cystadenomas $(\mathrm{n}=5)$, borderline-tumors $(\mathrm{n}=19)$, primary invasive carcinomas $(\mathrm{n}=178)$ and recurrent carcinomas $(\mathrm{n}=11)$; (C) correlation of high CDH11 expression (upper 75\% = quartiles 2-4) with advanced stage; (D) correlation of high CDH11 expression with nodal involvement. FIGO, International Federation of Gynecology and Obstetrics.

subgroups, there was a non-significant tendency showing lower CDH11 expression in endometrioid carcinomas compared with serous tumors (data not shown). No other significant associations with clinical or histological parameters were observed in this group. By Kaplan-Meier analysis and log-rank test using different CDH11 cut-off values, no significant correlation with recurrence-free or overall survival was identified (data not shown).

Localization of $\mathrm{CDH} 11$ protein expression detected by immunohistochemistry. Since CDH11 expression had been reported not only in tumor cells, but also in stromal or endothelial cells, we analyzed representative tumors from each group (8 invasive carcinomas, 12 borderline tumors and 3 cystadenomas) for CDH11 expression by immunohistochemistry. Strikingly, invasive carcinoma cells were either CDH11-negative or displayed only weak nuclear or cytoplasmic CDH11 expression. We did not detect any membranous CDH11 staining in tumor cells. By contrast, strong CDH11 staining was observed in the neighboring vessel walls and stromal matrix (Fig. 3). The comparison with immunohistochemical detection of the endothelial marker CD31 in parallel sections demonstrated CDH11 expression in endothelia and vascular smooth muscle cells around the blood vessels (Fig. 3C). Isotypic controls were always negative (data not shown).

\section{Discussion}

The function of CDH11 attracted interest during our prior experimental studies, in which decreased adhesive properties of ovarian cancer cells upon c-Fos overexpression were demonstrated, accompanied by downregulation of various adhesion proteins, including CDH11. Based on the results from other tumor entities and the first data on ovarian tumors (11), we investigated CDH11 mRNA and protein expression in a large cohort of ovarian carcinomas, borderline tumors and benign cystadenomas. By western blot analysis and RT-qPCR, we detected decreasing CDH11 expression with increasing malignancy, with the highest expression observed in benign tumors. By immunohistochemistry we demonstrated that, within the tumor tissue, CDH11 was predominantly expressed in blood vessel walls and stromal extracellular matrix components. This finding suggests that the difference in CDH11 expression between benign, borderline and malignant tumors, as demonstrated by RT-qPCR and western blot analysis, may be attributed to the different tumor-stroma ratios, with the largest fraction of stromal cells found in benign tumors.

CDH11 expression in myofibroblasts or vascular smooth muscle cells has been previously reported $(15,16)$, where it promotes cell proliferation and migration. Upregulation of CDH11 expression in differentiated myofibroblasts was 

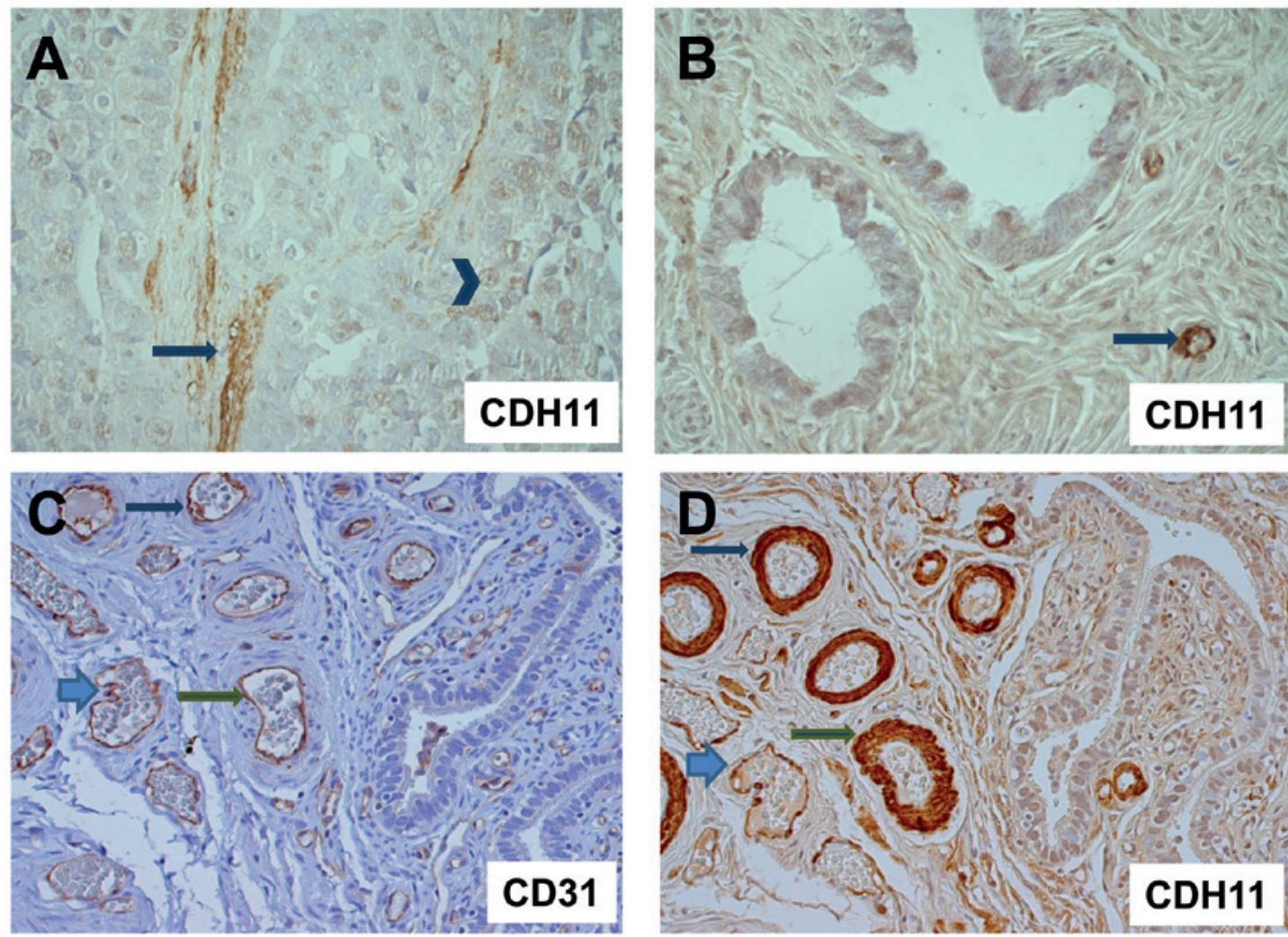

Figure 3. Cadherin-11 (CDH11) immunohistochemistry. (A) Invasive serous carcinoma exhibiting stromal immunoreactivity (arrow) and weak nuclear staining in epithelial tumor cells (arrowhead); magnification, x400; (B) ovarian cystadenoma with CDH11-positive capillaries (arrow) and negative or weak staining of epithelial cells; magnification, x400. (C) Borderline tumor with CD31 staining for blood vessels and (D) parallel section with CDH11 staining, exhibiting strong immunoreactivity of endothelial cells and vascular smooth muscle cells around blood vessels (arrows); magnification, $\mathrm{x} 200$. The corresponding structures in $\mathrm{C}$ and $\mathrm{D}$ are indicated by identical arrow types.

detected during dermal wound healing (15), where it contributed to cell contraction. Interestingly, CDH11-mediated cell-cell contacts in fibroblasts lead to strong upregulation of vascular endothelial growth factor-D expression, indicating that it may be involved in the angiogenic process (17). The CDH11 overexpression in fibroblasts in the vicinity of tumor cells concurs with the observation that the tumor microenvironment shares several characteristics with a chronic wound $(18,19)$.

In contrast to stromal cells, we did not identify any membranous CDH11 expression in epithelial ovarian tumor cells of different malignancy, but only weak or absent cytoplasmic or nuclear immunoreactivity. A similar staining pattern was reported in glioma cells, where an anti-invasive function of CDH11 was hypothesized (20).

Regarding the role of CDH11 in human malignant tumors, conflicting results were reported: A tumor suppressor function of CDH11 expression was reported in retinoblastomas (21) and gliomas (20), whereas high CDH11 expression correlated with a more malignant subtype in colorectal cancer (22), prostate cancer (8), breast cancer cell lines (10) and osteosarcomas (23). The latter immunohistochemical study is in contrast to an RT-PCR-based investigation, which reported a correlation of CDH11 expression with a good prognosis in this tumor type (24). In the light of our results, this contradiction may be associated with methodical differences.

In ovarian tumors, CDH11 mRNA expression was previously reported to be upregulated in metastatic lesions compared to primary tumors (11). In the present study, high CDH11 protein expression, as shown by western blot analysis, was associated with advanced stage and nodal involvement, which points to the same direction. Since the tumor-stroma ratio does not differ in carcinomas of different stage or nodal status, the RT-qPCR or western blot analysis results are comparable within this tumor group. However, although these correlations suggest a possible oncogenic role of this adhesion molecule in ovarian cancer, the corresponding follow-up data show that CDH11 expression is not significantly associated with prognosis in ovarian carcinomas.

In conclusion, to the best of our knowledge, this is the first study of CDH11 expression in a large cohort of ovarian tumors of different histological subtypes. Among ovarian tumors, CDH11 is predominantly expressed in stromal cells (myofibroblasts, vascular smooth muscle cells and endothelial cells), leading to the highest expression in benign tumors with a high stromal fraction. Although in malignant ovarian cancer, high CDH11 expression correlated with characteristics associated with an unfavorable outcome, the lack of a prognostic significance in the survival analysis indicates that it is not a suitable marker or therapeutic target in this type of tumor.

\section{Acknowledgements}

We would like to thank Maila Rossberg and Kathrin Eylmann for their excellent technical assistance. 


\section{References}

1. Berx G and van Roy F: Involvement of members of the cadherin superfamily in cancer. Cold Spring Harb Perspect Biol 1: a003129, 2009.

2. Davidson B, Berner A, Nesland JM, et al: E-cadherin and alpha-, beta-, and gamma-catenin protein expression is up-regulated in ovarian carcinoma cells in serous effusions. J Pathol 192: 460-469, 2000.

3. Sundfeldt K, Piontkewitz Y, Ivarsson K, et al: E-cadherin expression in human epithelial ovarian cancer and normal ovary. Int J Cancer 74: 275-280, 1997.

4. Hazan RB, Kang L, Whooley BP and Borgen PI: N-cadherin promotes adhesion between invasive breast cancer cells and the stroma. Cell Adhes Commun 4: 399-411, 1997.

5. Qian X, Anzovino A, Kim S, et al: N-cadherin/FGFR promotes metastasis through epithelial-to-mesenchymal transition and stem/progenitor cell-like properties. Oncogene 33: 3411-3421, 2014.

6. Van Roy F: Beyond E-cadherin: Roles of other cadherin superfamily members in cancer. Nat Rev Cancer 14: 121-134, 2014.

7. Chu K, Cheng CJ, Ye X, Lee YC, Zurita AJ, Chen DT, Yu-Lee LY, Zhang S, Yeh ET, Hu MC, et al: Cadherin-11 promotes the metastasis of prostate cancer cells to bone. Mol Cancer Res 6 : 1259-1267, 2008.

8. Huang CF, Lira C, Chu K, et al: Cadherin-11 increases migration and invasion of prostate cancer cells and enhances their interaction with osteoblasts. Cancer Res 70: 4580-4589, 2010.

9. Nieman MT, Prudoff RS, Johnson KR and Wheelock MJ: $\mathrm{N}$-cadherin promotes motility in human breast cancer cells regardless of their E-cadherin expression. J Cell Biol 147: 631-644, 1999

10. Pishvaian MJ, Feltes CM, Thompson P, et al: Cadherin-11 is expressed in invasive breast cancer cell lines. Cancer Res 59: 947-952, 1999.

11. Bignotti E, Tassi RA, Calza S, et al: Gene expression profile of ovarian serous papillary carcinomas: identification of metastasis-associated genes. Am J Obstet Gynecol 196: 245.e1-11, 2007.

12. Oliveira-Ferrer L, Rößler K, Haustein V, et al: c-FOS suppresses ovarian cancer progression by changing adhesion. Br J Cancer 110: 753-763, 2014.

13. Mahner S, Baasch C, Schwarz J, et al: C-Fos expression is a molecular predictor of progression and survival in epithelial ovarian carcinoma. Br J Cancer 99: 1269-1275, 2008.
14. Schröder C, Schumacher U, Müller V, et al: The transcription factor Fra-2 promotes mammary tumour progression by changing the adhesive properties of breast cancer cells. Eur J Cancer 46: 1650-1660, 2010

15. Hinz B, Pittet P, Smith-Clerc J, Chaponnier C and Meister JJ: Myofibroblast development is characterized by specific cell-cell adherens junctions. Mol Biol Cell 15: 4310-4320, 2004.

16. Monahan TS, Andersen ND, Panossian H, et al: A novel function for cadherin 11/osteoblast-cadherin in vascular smooth muscle cells: Modulation of cell migration and proliferation. J Vasc Surg 45: 581-589, 2007.

17. Orlandini $M$ and Oliviero S: In fibroblasts Vegf-D expression is induced by cell-cell contact mediated by cadherin-11. J Biol Chem 276: 6576-6581, 2001.

18. Arnold KM, Opdenaker LM, Flynn D and Sims-Mourtada J: Wound healing and cancer stem cells: Inflammation as a driver of treatment resistance in breast cancer. Cancer Growth Metastasis 8: 1-13, 2015.

19. Dvorak HF: Tumors: Wounds that do not heal. Similarities between tumor stroma generation and wound healing. N Engl J Med 315: 1650-1659, 1986.

20. Delic S, Lottmann N, Jetschke K, Reifenberger G and Riemenschneider MJ: Identification and functional validation of CDH11, PCSK6 and SH3GL3 as novel glioma invasion-associated candidate genes. Neuropathol Appl Neurobiol 38: 201-212, 2012.

21. Marchong MN, Yurkowski C, Ma C, Spencer C, Pajovic S and Gallie BL: Cdh11 acts as a tumor suppressor in a murine retinoblastoma model by facilitating tumor cell death. PLoS Genet 6: e1000923, 2010.

22. De Sousa E Melo F, Wang X, Jansen M, et al: Poor-prognosis colon cancer is defined by a molecularly distinct subtype and develops from serrated precursor lesions. Nat Med 19: 614-618, 2013.

23. Deng Z, Niu G, Cai L, Wei R and Zhao X: The prognostic significance of CD44V6, CDH11, and $\beta$-catenin expression in patients with osteosarcoma. BioMed Res Int 2013: 496193, 2013.

24. Nakajima G, Patino-Garcia A, Bruheim S, et al: CDH11 expression is associated with survival in patients with osteosarcoma. Cancer Genomics Proteomics 5: 37-42, 2008. 